https://doi.org/10.5719/aub-g/70.1/4

\title{
L'ÉVALUATION, UNE ÉTAPE IMPORTANTE DANS L'ÉTUDE DES GÉOSITES. APPLICATIONS AU SITE FIERBĂTORILE DE BERCA, VALLÉE DE BUZĂU
}

\author{
GABRIEL COSMIN ILIE ${ }^{1}$, FLORINA GRECU1
}

\begin{abstract}
Résumé
Cet article traite de l'importance de l'étape d'évaluation en tant que partie distincte de l'étude des géosites en appliquant une méthode d'évaluation résultant des caractéristiques géographiques d'un site. À cet égard, le relief créé par les volcans boueux de Berca (également connus sous le nom de Fierbătorile de Berca) est analysé, en soulignant la valeur scientifique sur la base des indicateurs suivants: l'intégrité du géosite (stade de conservation, degré d'influence à la fois des facteurs naturels et de l'intervention anthropique), la représentativité (exemplarité du site par rapport à l'espace de référence), la rareté, l'importance pédagogique et la valeur géohistorique. La représentativité et la rareté du paysage/site nécessitent la prise en compte et l'analyse de valeurs supplémentaires, telles que les valeurs écologiques, esthétiques, culturelles.

Fierbătorile de Berca, élément de géopatrimoine représentatif pour les Subcarpates de Courbure, mais aussi pour l'ensemble du territoire de la Roumanie, sont peu étudiées à cet égard dans la littérature spécialisée. L'objectif de l'étude vise l'importance de connaître ce géosite à partir nécessairement de la valeur scientifique, y compris la valeur éducative institutionnalisée ou touristique.
\end{abstract}

Mots clés : géosite, évaluation, Buzău, volcans de boue, Berca

\section{Introduction}

Dans la communauté géomorphologique, de nouvelles pistes d'étude du patrimoine géologique et culturel ont été synthétisées en utilisant des termes tels que géotope, géosite ou géomorphosite

1 Université de Bucarest, Faculté de Géographie, 1 Bd. N. Bălcescu, sector 1, 010041 Bucarest, Roumanie. E-mail: gabriel.ilie@drd.unibuc.ro, grecu@geo.unibuc.ro 
(Panizza, 2001). Les géosites sont des éléments à la surface de la terre ayant acquis une valeur scientifique, culturelle-historique, esthétique et même socio-économique, en raison de leur perception ou leur l'évaluation par l'homme (Reynard et al, 2007). Ceux-ci ont constitué un élément de nouveauté dans l'approche de l'étude de la géographie, de sorte qu'au cours des deux dernières décennies, un nombre croissant de chercheurs ont mené des études approfondies sur la définition, l'inventaire, la cartographie, l'évaluation, la conservation et la valorisation supérieure des géosites.

Puisque la perception humaine peut prendre des nuances subjectives lorsqu'on considère la hiérarchisation de l'importance des différentes valeurs ou l'appréciation de chaque valeur, les efforts des géographes se sont orientés vers la proposition de méthodes standards d'évaluation des géosites afin d'être en mesure de faire une appréciation plus réaliste des valeurs qu'ils possèdent. En ce sens, la littérature internationale propose une série de modèles d'évaluation pertinents, dont la méthode proposée par Mario Panizza qui considère l'évaluation des géomorphosites selon des critères scéniques, socio-économique, culturel et scientifique (Reynard, 2009) ou la méthode d'évaluation de la valeur globale des géosites, qui traite la valeur scientifique séparément des autres additionnelles (Reynard, 2006). D'autres méthodes d'évaluation des géosites incluent la méthode proposée par les Universités de Moderna et Regio Emilia, qui ne met en évidence que la valeur scientifique des géosites (Coratza et Giusti, 2005; Giusti, 2012) ou la méthode proposée par l'Université de Cantabrie, qui évalue la valeur scientifique, le potentiel d'utilisation, les menaces potentielles et les mesures de protection nécessaires (Bruschi et Cendrero, 2005), ainsi que les méthodes de recherche utilisées par les universités roumaines, qui mettent l'accent sur les valeurs scientifiques, esthétiques et pratiques-touristiques.

Dans la littérature scientifique roumaine, la question des géosites a suivi les pistes de recherche et les méthodes d'analyse proposées par la littérature internationale au début des années 2000 et s'est surtout développée au cours de la dernière décennie, les principaux contributeurs étant les professeurs de l'Université de Bucarest et de l'Université de Oradea. Des études ont été menées qui suivent à la fois des aspects théoriques concernant les géosites (Ilieş et Josan 2008, 2009) 
et leur inventaire (Comănescu, 2008), ainsi que des recherches pratiques d'inventaire des géosites dans certaines régions du pays, souligner l'importance de critères significatifs pour la région, telles que les Monts Apuseni (Ilieş et Josan, 2007), les Monts Bucegi (Comănescu et Nedelea, 2010), les Monts Făgăraş (Comănescu et al, 2011), le Défilé du Danube (Grecu et Iosif, 2014a, 2014b) ou la Dobroudja du Sud (Rădulescu et Grecu, 2017). Les préoccupations d'inscrire différents éléments et processus géomorphologiques au sein du patrimoine naturel de la Roumanie ont conduit à de nouvelles approches de la recherche du relief, dans la vision d'une utilisation pratique pluridisciplinaire (Reynard et al, 2018, Grecu et al, 2019).

De nombreuses autres méthodes ont été développées ces dernières années par des chercheurs du monde entier, adaptées soit aux éléments d'identité physique et géographique des régions analysées, soit aux catégories de valeurs à évaluer, ou lors de l'utilisation de la technique informatisée dans l'analyse. Les géosites, respectivement les géomorphosites, sont le résultat de l'action de processus géomorphologiques sur la croûte terrestre, ce sont donc des éléments de la dynamique du relief. A ce titre, ils constituent la «cellule de base » des classifications et hiérarchies visant à la protection et à l'aménagement du territoire. La multitude de critères utilisés notamment dans l'identification et l'évaluation des sites, moins dans leur dynamique et leur évolution (Grecu, 2017), met en discussion la position subjective de l'auteur et le rapport à la réalité territoriale. L'étude des géosites fait partie de la dynamique terrestre. Avec d'autres notions (paysage, géodiversité, géoécologie, etc.), elle renvoie à la partie appliquée et socialement liée de la nature (Comănescu et al, 2015-2016, Reynard et al, 2018).

\section{Zone d'étude}

La rivière de Buzău prend sa source dans les Monts Ciucaş et recueille les eaux d'une superficie de $5264 \mathrm{~km}^{2}$ (Ujvari, 1972, Mocanu-Vargancsik et Bărbulescu, 2018). La rivière traverse les comtés de Braşov, Covasna, Buzău, Brăila et 116 unités administrativesterritoriales (Popa et al, 2019). La composition lithologique et la dynamique de la vallée de Buzău en zone de montagne ont imposé 
la sectorisation du cours d'eau en 4 subdivisions (Grecu, 2018), chacune d'elles étant individualisée par le potentiel géomorphologique complexe. Entre les localités Vama Buzăului et Întorsura Buzăului, la rivière sépare les Monts Buzău (sur la rive droite) des Monts Teleajen (sur la rive gauche). En aval de la localité de Întorsura Buzăului, la rivière suit un tracé à travers les Monts Buzău, qu'elle traverse en aval jusqu'à la localité de Nehoiu, où elle pénètre dans les Subcarpates de Buzău et sépare Dealul Cornetului (sur la rive droite) de Culmea Ivănețu et Dealul Blidisel (sur la rive gauche) vers la zone de la dépression Cislău, afin d'enregistrer un changement de la direction d'écoulement, vers l'est, à travers le nord de la colline Ciolanu, jusqu'à atteindre la Plaine Roumaine (Institutul de Geografie, 1987, 1992).

Les caractéristiques géomorphologiques de la vallée sont sujettes à des changements lors de l'entrée dans l'espace subcarpatique, car la composition lithologique devient dominée par des roches à faible degré de dureté. L'augmentation de l'ouverture de la vallée, la diminution de la pente des versants, l'augmentation significative de la largeur du lit de la rivière ainsi que l'individualisation de la plaine inondable sont des aspects qui sont dus à la genèse et à l'évolution de la rivière dans un espace à la géologie et à la tectonique spécifiques à la zone de courbure de Vrancea (Visarion et al, 1977). Les dépôts sédimentaires du Myo-Pliocène et du Quaternaire moins résistants à l'érosion ont favorisé l'apparition de processus de pente et de lit de rivière, tels que glissements de terrain, érosion de pente et de lit de rivière, accumulations, méandres, etc (Institutul de Geografie, 1987, 1992; Ielenicz, 1984).

La genèse des Subcarpates de Buzău, influencée par le contact entre la courbure des Carpates et la Plate-forme Moesique, est responsable de la composition lithologique complexe de la région. La présence des aires avec des volcans de boue dans les Subcarpates de Buzău est une preuve de pressions tectoniques, fournissant des indications importantes sur la structure géologique ainsi que sur l'activité sismique. L'arrangement des couches sédimentaires est affecté par certaines dislocations développées à la fois longitudinalement et transversalement, et l'apparition d'éruptions boueuses en surface marque l'intersection de telles fractures, l'alignement de cônes indiquant pratiquement la direction des failles (Frunzescu et Brănoiu, 2004). 


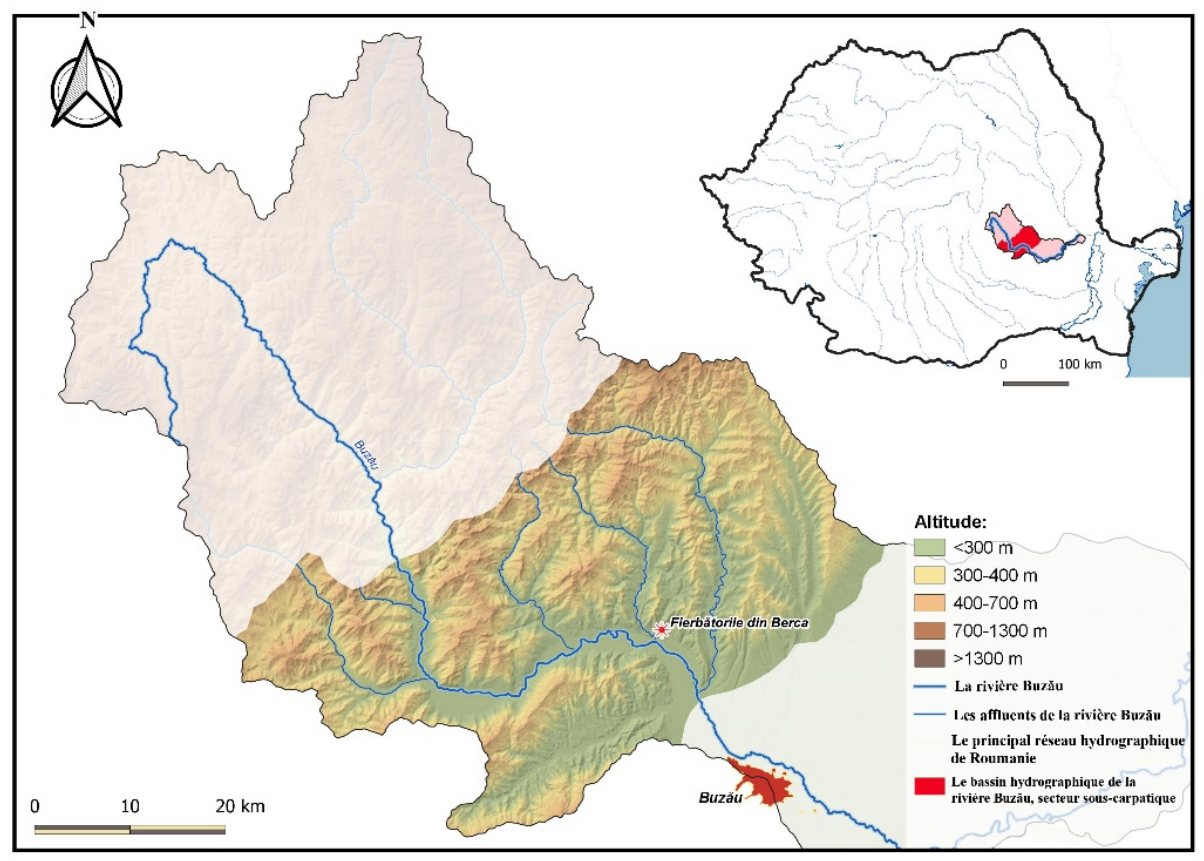

Fig. 1. Situation géographique de Fierbătorile de Berca dans le bassin hydrographique de la rivière de Buzău

L'aire connue sous le nom de Fierbătorile de Berca est située dans le secteur subcarpatique de la vallée de la rivière de Buzău (Fig. 1), sur le territoire administratif de la commune de Berca. Bien qu'elle n'ait pas actuellement le statut de zone protégée, c'est l'une des régions les plus représentatives avec des volcans de boue en Roumanie (à côté de Pâclele Mari, Pâclele Mici et les volcans de boue de Beciu), réunissant des formations créées par l'expulsion des boues, de méthane, de dioxyde de carbone et d'azote (Baciu et al, 2007) dans certaines conditions lithologiques.

La région a été étudiée pour la première fois dans la littérature au XIXe siècle (Coquand, 1867), et les préoccupations de recherche se sont progressivement focalisées sur des questions telles que l'analyse des gaz 
dans le matériau boueux (Costăchescu, 1906), la relation entre la présence de volcans de boue et l'accumulation d'hydrocarbures en sous-sol (Ciocârdel, 1949), l'analyse stratigraphique de la région de Berca (Macarovici, 1961), la classification morphologique des cônes volcaniques de Berca (Sencu, 1985), l'analyse de la relation entre l'intensité des émissions de gaz et la sismicité de la région de courbure (Baciu et Etiope, 2005).

Fierbătorile de Berca présente, de par leur nature, des caractéristiques remarquables qu'il convient d'évaluer par une méthode spécialement conçue pour mettre en évidence la pertinence scientifique, le contexte éducatif réuni, mais aussi la contribution que le site apporte à l'identité géomorphologique de la vallée de Buzău.

\section{Méthodologie appliquée}

Le processus d'étude de l'élément de géopatrimoine Fierbătorile de Berca s'est déroulé en plusieurs étapes, partant de l'analyse du matériel bibliographique existant (caractéristiques géomorphologiques et lithologiques de l'aire, la carte géologique, les images satellites) et se poursuivant par des sorties sur le terrain pour une bonne observation des processus en cours.

Pour établir l'importance du site (Tableau 1), nous avons suivi le modèle d'évaluation des géosites en Roumanie (Iosif, 2014) basé sur la méthode d'évaluation proposée par le professeur Emmanuel Reynard de l'Institut de Géographie de l'Université de Lausanne, ses études dans le domaine des géosites étant considérées comme un repère important dans la littérature scientifique internationale. Selon sa vision, les géosites sont "des portions de la surface terrestre d'une importance particulière pour la compréhension de l'évolution de la Terre" (Reynard, 2009) et leur valeur principale est scientifique, tandis que d'autres ensembles de valeurs (culturelle, économique, esthétique, écologique) sont considérées comme secondaires. 
Tableau 1

Modèle proposé par D. Iosif pour l'évaluation des sites en Roumanie adapté de la version originale de E. Reynard

\begin{tabular}{|l|l|}
\hline \multirow{4}{*}{ Valeur du géosite } & \multicolumn{1}{|c|}{$\begin{array}{c}\text { Critères permettant d'apprécier } \\
\text { la valeur }\end{array}$} \\
\hline \multirow{4}{*}{ Scientifique } & Intégrité \\
\cline { 2 - 2 } & Représentativité \\
\cline { 2 - 2 } & Rareté \\
\cline { 2 - 2 } & Éducation \\
\cline { 2 - 2 } Écologique & Géohistoire \\
\hline \multirow{4}{*}{ Esthétique } & Influence écologique \\
\cline { 2 - 2 } & Protection du site \\
\hline \multirow{4}{*}{ Culturelle } & Points d'observation \\
\cline { 2 - 2 } & $\begin{array}{l}\text { Contraste et développement } \\
\text { vertical }\end{array}$ \\
\hline \multirow{2}{*}{ Économique } & Importance religieuse \\
\cline { 2 - 2 } & Importance historique \\
\cline { 2 - 2 } & Importance artistique \\
\hline
\end{tabular}

Pour apprécier l'importance scientifique, la méthode d'évaluation propose les indicateurs suivants : l'intégrité du géosite (l'état de conservation, le degré d'influence à la fois des facteurs naturels et de l'intervention anthropique) la représentativité (exemplarité du site par rapport à l'espace de référence), la rareté, l'importance éducative et la valeur géohistorique.

Les valeurs additionnelles analysées sont : la valeur écologique (appréciée selon l'influence écologique et la protection du site), la valeur esthétique (appréciée selon les points d'observation, le contraste et le développement vertical), la valeur culturelle (appréciée selon l'importance 
religieuse, l'importance historique, l'importance littéraire et artistique), la valeur économique (appréciée selon la production économique).

La quantification de tous les critères d'évaluation a été réalisée en donnant les scores suivants : 0 (nulle), 0.25 (valeur faible), 0.50 (valeur moyenne), 0.75 (valeur élevée) et 1 (valeur très élevée), et pour calculer la moyenne obtenue par une valeur, la somme des scores par critère est divisée par le nombre de critères. Par exemple, afin d'évaluer la valeur scientifique, on va procéder à faire la moyenne arithmétique entre les scores obtenus par les critères d'intégrité, de représentativité, de rareté, d'éducation et de géohistoire. La moyenne obtenue par chaque valeur sera donc une valeur comprise entre 0 et 1 .

Valeur scientifique $=\frac{\text { Integrité }+ \text { Représentativité }+ \text { Rareté }+ \text { Éducation }+ \text { Géohistoire }}{5}$

Valeur écologique $=\frac{\text { Influence écologique }+ \text { Protection du site }}{2}$

Valeur esthétique $=$ Points d'observation + Contraste et développement vertical

3

Val. culturelle $=$ Importance religieuse + Importance historique + Importance artistique 3

La valeur globale du géosite sera calculée à la fin, selon la méthode, comme une moyenne pondérée entre les scores de toutes les valeurs, faisant mention que la valeur scientifique aura un double coefficient par rapport aux valeurs additionnelles: 
$\mathrm{V} \cdot$ globale $=\frac{2 \cdot \mathrm{V} . \text { scientifique }+ \text { V.culturelle }+ \text { V.esthétique }+ \text { V.écologique }+ \text { V.économique }}{6}$

\section{Résultats et discussions}

\subsection{Description du site}

Les volcans de boue sur le territoire de la commune de Berca, compté de Buzău sont les sites les plus représentatifs de ce type en Roumanie et en même temps l'un des sites les plus importants avec de pertinence lithologique et géomorphologique situé le long de la vallée de la rivière de Buzău. Ils sont présents dans les collines des Subcarpates sous la forme de 4 zones distinctes: Pâclele de la Beciu, Pâclele Mari, Pâclele Mici et Fierbătorile de la Berca.

L'aire la plus méridionale avec des volcans de boue dans les Subcarpates de Courbure est connue sous le nom de Fierbătorile de Berca et se situe dans la partie nord-est du village-résidence de Berca, à environ $2 \mathrm{~km}$ en droite ligne du lit de la rivière de Buzău, dans une région collinaire où les altitudes ne dépassent pas $280 \mathrm{~m}$ (Tableau 2). La région est très bien entourée par une végétation mixte (Fig. 2).

Tableau 2

Fierbătorile de Berca, données générales

$\begin{array}{ll}\text { Coordonnées géographiques } & 45^{\circ} 17^{\prime} 47^{\prime \prime} \mathrm{N} 26^{\circ} 41^{\prime} 39^{\prime \prime} \mathrm{E} \\ \text { Type de géosite } & \text { Polygonal } \\ \text { Altitude minimale } & 205 \text { mètres } \\ \text { Altitude maximale } & 280 \text { mètres } \\ \text { environ } 5 \text { ha } \\ \text { Type de propriété }\end{array} \quad \begin{aligned} & \text { publique, village de Berca, } \\ & \text { compté de Buzău } \\ & \text { géomorphologique, lithologique } \\ & \text { Centres d'intérêt }\end{aligned}$




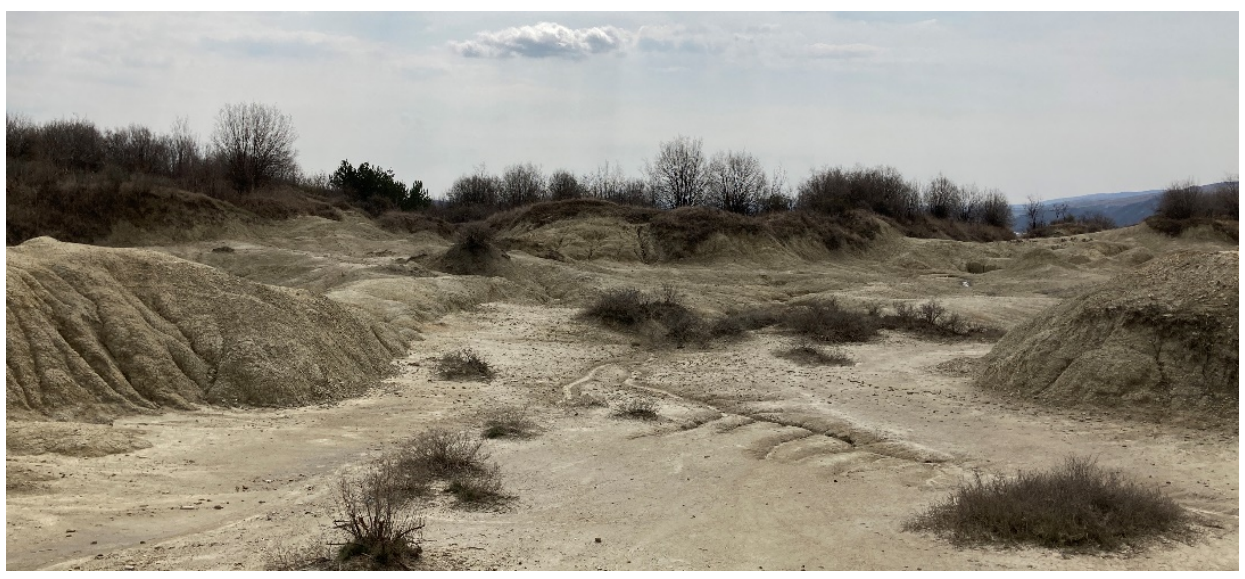

Fig. 2. Le plateau nord dans la région de Fierbătorile de Berca, bordé de végétation de feuillus et mélange

Fierbătorile réunissent deux plateaux situés à une distance d'environ 400 mètres l'un de l'autre. Le plateau nord a une plus grande ouverture d'environ 2,7 hectares et il est traversé par des pipelines de combustibles fossiles, dont l'agencement a également facilité l'accès direct au plateau. Le plateau sud a une superficie plus petite (environ 2 hectares) et il n'est relié par aucun accès à celui du nord, donc pour y arriver il faut parcourir une distance de 400 mètres sur un terrain accidenté.

Le nombre de cratères actifs est relativement faible dans le cas des deux plateaux, mais il existe de nombreux cônes éteints qui soutiennent le développement d'espèces végétales (Fig. 3), comme l'obione (Obione verrucifera). Le relief des deux plateaux a été intensément façonné par les anciennes éruptions et le ruissellement inorganisé des eaux de pluie, dans le contexte d'absence de végétation et d'un sol riche en argile. Les vieux cônes montrent des traces de ruissellement à la surface à travers lesquelles l'eau et le matériau érodé sont dirigés vers les canaux de drainage et vers les ravines.

\subsection{Morphogenèse}

Fierbătorile de Berca (Mares de boues de Berca) sont des formes de relief pseudo-volcanique qui sont apparues par l'épanchement de boue et son accumulation subséquente, par la réunion cumulative de conditions 
lithologiques spécifiques: la présence dans le sous-sol de gisements de combustibles fossiles sous pression lithostatique, l'apparition de fissures dans les dépôts sédimentaires situés au-dessus des gisements de gaz, la présence d'une couche uniforme d'argile près de la surface de la terre ainsi que la présence d'eau dans les horizons aquifères captifs (Achim, 2016).
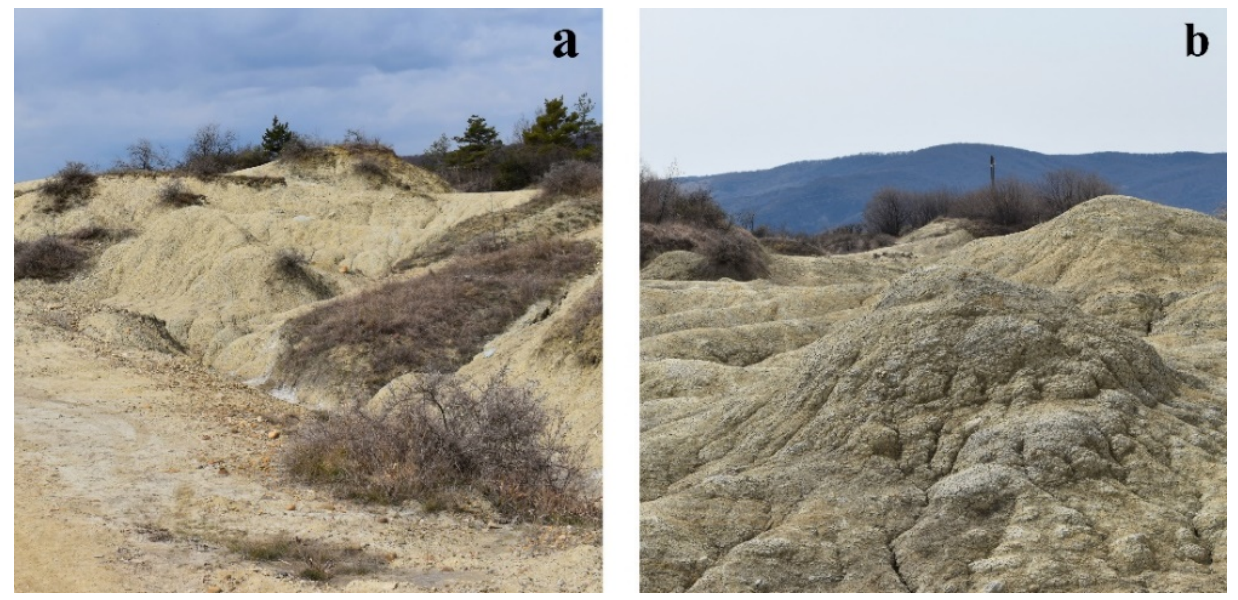

Fig. 3. Formes de relief façonnées par d'anciennes éruptions boueuses. a. cônes qui abritent des espèces végétales halophiles, par exemple l'obione (Obione verrucifera)

b. cônes façonnés par le ruissellement inorganisé des eaux de pluie, dans le contexte d'absence de végétation.

L'aire chevauche l'une des plus anciens champs de pétrole du pays, et la présence de mares de boues (fierbători) est directement liée à l'existence de ce gisement qui, suite à des processus de dégradation tectonique, communique avec la surface de la terre. La genèse des volcans de boue implique l'apparition d'un contact entre le gisement de combustibles fossiles (sous pression lithostatique), la couche d'argile et les horizons aquifères, se manifestant en repoussant l'eau et l'argile vers la surface par la remontée des gaz (Frunzescu et Brănoiu, 2004). En remontant vers la surface, les fractions gazeuses absorbent l'eau infiltrée 
ainsi que les matériaux argileux qu'elles déposent en surface dans les régions des cratères.

Dans les deux plateaux qui forment Fierbătorile de Berca, les éruptions actuelles génèrent une très faible quantité de matériau solide, le degré de fluidité étant extrêmement élevé. Pour cette raison, les zones d'éruptions actives n'ont pas de formes coniques typiques telles que celles des volcans de boue de Beciu, Pâclele Mari ou Pâclele Mici (Fig. 4).

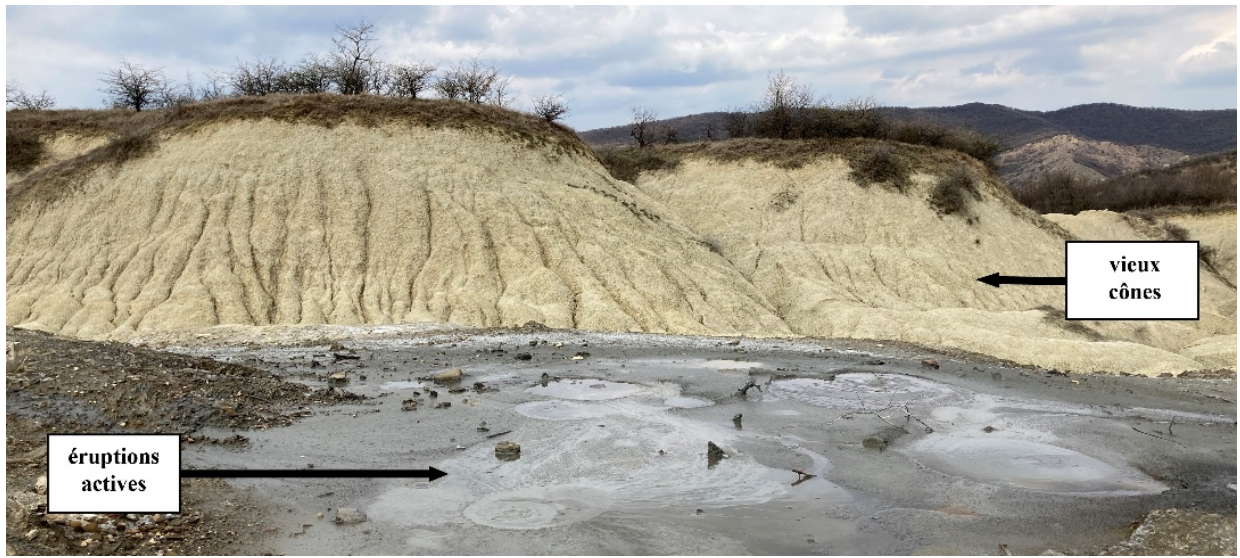

Fig. 4. Différences entre les éruptions actuelles, où les cratères apportent en surface un matériau très fluide et les anciens cônes, formés sur la base d'un matériau plus riche en argile.

À l'heure actuelle, le matériau boueux arrive en surface dans de petits plateaux éruptifs contenant des cratères sous la forme de dépressions rondes et lisses, où une grande partie du matériau enlevé reste dans le cratère et, au fur et à mesure qu'il reçoit de nouvelles émissions de boue et de gaz, il s'écoule lentement, mais ne conduit pas à l'accumulation du matériau en forme de cônes.

\section{3 Évaluation des valeurs du site „Fierbătorile de Berca”}

En appliquant le modèle proposé par D. Iosif pour l'évaluation des géosites en Roumanie, on a obtenu les scores et les résultats suivants (tableau 3): 
Tableau 3

\section{Évaluation des valeurs du géosite Fierbătorile de Berca}

\begin{tabular}{|l|l|c|}
\hline \multicolumn{3}{|c|}{ Valeur scientifique } \\
\hline Critère évalué & \multicolumn{1}{|c|}{ Commentaires } & Score \\
\hline Intégrité & $\begin{array}{l}\text { Le site n'a subi aucune dégradation en } \\
\text { raison de processus d'érosion ou } \\
\text { d'interventions anthropiques. L'aire des } \\
\text { volcans de boue est intacte. }\end{array}$ & 1 \\
\hline Représentativité & $\begin{array}{l}\text { Le site met en évidence certaines des } \\
\text { principales caractéristiques de la } \\
\text { géomorphologie de la région. La genèse } \\
\text { des éruptions boueuses s'explique par la } \\
\text { présence du champ de pétrole de Berca - } \\
\text { Beciu - Arbănaşi, par la composition } \\
\text { lithologique qui comprend des dépôts } \\
\text { marno-argileux, ainsi que par une certaine } \\
\text { circulation des eaux souterraines. }\end{array}$ & \\
\hline Rareté & $\begin{array}{l}\text { Le site présente des dimensions, une } \\
\text { lithologie et des caractéristiques } \\
\text { remarquables dans l'espace de référence. }\end{array}$ & 0,75 \\
\hline Éducation & $\begin{array}{l}\text { Par son intégrité, par la représentativité } \\
\text { des caractéristiques géomorphologiques } \\
\text { mais aussi par sa rareté, le site répond à } \\
\text { un contexte éducatif remarquable. }\end{array}$ & 0,75 \\
\hline Importance religieuse & $\begin{array}{l}\text { Le site est connu au niveau national pour } \\
\text { des manifestations éruptives, ce qui } \\
\text { certifie la présence de combustibles } \\
\text { fossiles dans le sous-sol et démontre les } \\
\text { conditions lithologiques et tectoniques qui } \\
\text { permettent l'apparition en surface de } \\
\text { volcans de boue. }\end{array}$ & 0,75 \\
\hline Importance historique & $\begin{array}{l}\text { Le site n'a aucune importance religieuse. } \\
\text { Géohistoire }\end{array}$ & 0 \\
\hline
\end{tabular}




\begin{tabular}{|c|c|c|}
\hline Importance artistique & $\begin{array}{l}\text { Le site présente des caractéristiques esthétiques } \\
\text { particulières, résultant de la manifestation } \\
\text { de processus géomorphologiques tels que } \\
\text { des plateaux ayant des éruptions boueuses, } \\
\text { des formes d'érosion causées par un } \\
\text { ruissellement inorganisé des eaux } \\
\text { (ruissellement diffus, rigoles, ravines), des } \\
\text { efflorescences salines développées pendant } \\
\text { les périodes sèches, des volcans de boue } \\
\text { éteints mais aussi actifs. }\end{array}$ & 0,25 \\
\hline \multicolumn{3}{|c|}{ Valeur écologique } \\
\hline Critère évalué & Commentaires & Score \\
\hline Influence écologique & $\begin{array}{l}\text { Le site est un habitat non exclusif } \\
\text { d'espèces végétales déclarées monument } \\
\text { naturel: la nitraire (Nitraria schoberri) et } \\
\text { l'obione (Obione verrucifera). }\end{array}$ & 0,75 \\
\hline $\begin{array}{l}\text { Protection } \\
\text { du site }\end{array}$ & $\begin{array}{l}\text { Le site n'est pas inclus dans la liste des } \\
\text { aires protégées. }\end{array}$ & 0 \\
\hline \multicolumn{3}{|c|}{ Valeur esthétique } \\
\hline Critère évalué & Commentaires & Score \\
\hline Points d'observation & $\begin{array}{l}\text { Le site offre plusieurs points d'observation, } \\
\text { mais il est très bien masqué par des aires } \\
\text { forestières. }\end{array}$ & 0,25 \\
\hline Structure & $\begin{array}{l}\text { Le site s'étend sur une superficie d'env. } 5 \\
\text { hectares, et les contrastes présents dans } \\
\text { l'environnement sont concentrés sur le } \\
\text { site. }\end{array}$ & 1 \\
\hline \multicolumn{3}{|c|}{ Valeur économique } \\
\hline Critère évalué & Commentaires & Score \\
\hline Productivité & Le site ne génère aucun revenu & 0 \\
\hline
\end{tabular}

Le processus d'évaluation du site Fierbătorile de Berca a révélé un score de 0,80 pour la valeur scientifique. L'élément principal est la présence du relief pseudo-volcanique, apparu à la suite de l'épanchement de boue et de son accumulation subséquente, par la réunion cumulative de conditions lithologiques spécifiques. Le site se distingue par le degré 
extrêmement élevé de fluidité du matériau boueux remonté à la surface, c'est pourquoi, à l'heure actuelle, les éruptions n'ont pas de formes coniques typiques, mais de plateaux aplanis.

Parmi les valeurs additionnelles figurent la valeur écologique, soutenue par la présence d'espèces végétales halophiles endémiques et la valeur esthétique, soutenue par l'aménagement sur plusieurs hectares de mares de boues (fierbători), qui influence fortement le contraste du paysage. La valeur globale du site est de 0,44 (tableau 4).

Tableau 4

\section{La valeur globale du géosite Fierbătorile de Berca}

\begin{tabular}{|l|l|l|}
\hline \multicolumn{2}{|l|}{ Fierbătorile de Berca - valeur globale } \\
\hline Valeurs constitutives & Score & Coefficient \\
\hline Valeur scientifique & 0,80 & 2 \\
\hline Valeur culturelle & 0,08 & 1 \\
\hline Valeur écologique & 0,37 & 1 \\
\hline Valeur esthétique & 0,62 & 1 \\
\hline Valeur économique & 0 & 1 \\
\hline TOTAL & $\mathbf{0 , 4 4}$ & \multicolumn{1}{|l}{} \\
\cline { 1 - 2 } &
\end{tabular}

\section{Conclusions}

Le rôle de l'étape d'évaluation dans l'étude des géosites joue un rôle important dans la recherche scientifique. L'application d'une méthode d'évaluation offre au chercheur une série de perspectives, parmi lesquelles la possibilité de connaître et de comprendre certains processus ou phénomènes géologiques et géomorphologiques qui ont contribué à la genèse du géosite évalué, de la forme de relief dans laquelle se trouve le géosite ou son cadre lithologique. Les résultats offerts par le processus d'évaluation peuvent représenter la base des futures activités de recherche, orientées vers la protection et la conservation, dans le contexte de la sensibilisation à l'importance du géosite et à la nécessité de le protéger. 
La présente recherche a mis en évidence les arguments scientifiques qui renforcent l'importance de protéger le site Fierbătorile de Berca, notamment en l'inscrivant sur la liste des monuments naturels, car l'importance scientifique révélée par le processus d'évaluation a démontré la rencontre d'un contexte d'étude géologique et géomorphologique particulier.

Le processus d'évaluation du site Fierbătorile de Berca a mis en évidence une série d'éléments d'une valeur scientifique particulièrement élevée, dictée d'une part par la présence de caractéristiques géomorphologiques rares, uniques dans l'espace de référence, et d'autre part par de nombreuses pistes de recherche futures possibles. Le site, par sa genèse et son évolution, témoigne du passé géologique de la région. Il démontre la présence de caractéristiques lithologiques spécifiques, telles que la présence de combustibles fossiles sous pression lithostatique, la présence de couches sédimentaires argileuses et de fissures transversales et longitudinales dans les couches, qui permettent la circulation de matériaux boueux vers la surface. Le site met également en évidence certains processus tels que l'effusion d'eau et d'argile sous l'action des gaz ascendants, et présente des caractéristiques distinctes par rapport aux aires de volcans de boue de Pâclele Mari et Pâclele Mici en termes de fluidité.

Les mares de boues (fierbătorile) rassemblent des plateaux éruptifs actifs en association avec des formes de relief de type de cône éteint, résultant d'éruptions anciennes, ce qui indique certains changements dans la manifestation du phénomène présudo-volcanique qui ont conduit à une fluidité accrue des matériaux expulsés. L'occurrence de ces changements peut être attribuée à la sismicité de la zone, y compris la possibilité que de nouveaux horizons aquifères puissent être recoupés par l'appareil pseudo-volcanique à la suite de tremblements de terre, de sorte que le volume d'eau dans le matériau circulant vers le haut à travers les fissures des dépôts sédimentaires argileux devient plus élevé, ayant pour conséquence qu'en surface arrive un mélange boueux avec un degré de fluidité beaucoup trop élevé pour pouvoir produire les dépôts en forme de cônes.

D'un point de vue écologique, l'évaluation a mis en évidence le fait que, de par sa nature et ses caractéristiques lithologiques, Fierbătorile de 
Berca représentent un habitat pour les espèces végétales halophiles déclarées monument naturel, telles que la nitraire (Nitraria schoberri) et l'obione (Obione verrucifera), le site présentant également une pertinence botanique.

Il est donc nécessaire d'inscrire le géosite évalué sur la liste des réserves naturelles protégées par la loi, car il a la même importance géologique et géomorphologique que les aires protégées situées dans son nord, et les différences de degré de fluidité des éruptions actuelles donnent son unicité en termes de morphologie des cratères. De plus, il est nécessaire que des pistes de recherche futures possibles soient déployées, notamment en ce qui concerne l'analyse de la vulnérabilité du géosite à la fois aux facteurs environnementaux et à l'interaction possible avec le facteur anthropique.

\section{BIBLIOGRAPHIE}

Achim, F. (2016). Geomorfologie, Editura Universitară, Bucureşti, 453p;

Baciu, C., Etiope, G. (2005). Mud volcanoes and seismicity in Romania. In: Martinelli G. \& Panahi B. \& Panahi B. (Eds.), Mud volcanoes, Geodynamics and Seismicity, NATO Sci. Ser. Earth Environ., 51: 77-88, Springer Verlag, Berlin;

Baciu, C., Caracausi, A., Etiope, G., Italiano, F. (2007). Mud volcanoes and methane seeps in Romania: main features and gas flux. Annals of Geophysics, 50 (4): 501-511;

Bruschi, V.M., Cendrero, A. (2005). Geosite evaluation. Can we measure intangible values? Il Quaternario 18(1):293-306;

Ciocârdel, R. (1949). Regiunea petroliferă Berca-Beciu-Arbănaşi. Com. Geol., St. tehn.-econ., A1, 32 pp;

Comănescu, L. (2008). Definirea şi inventarierea geomorfositurilor, Comunicări de Geografie, volumul XII;

Comănescu, L., Nedelea, A. (2010). Analysis of some representative geomorphosites in the Bucegi Mountains: Between scientific evaluation and tourist perception. Area. 42. 406 416. 10.1111/j.1475-4762.2010.00937.x;

Comănescu, L., Nedelea, A., Dobre, R. (2011). Evaluation of geomorphosites in Vistea Valley (Fagaras Mountains-Carpathians, Romania). International journal of physical sciences. 6. 1161-1168;

Comănescu, L., Grecu F. (2015 - 2016). Geomorphosites and tourisms in Emmanuel Reynard coord. Intergeo-A digital platform for education to geomorphosites, Université de Lausanne www.univ.ch

Coquand, H. (1867). Sur le gîtes de pétrole de la Valachie et de la Moldavie et sur l'âge des terrains qui les contiennent. Bull. Soc. Géol. Fr., 2-ème Série, XXIV: 505-570; 
Coratza, P., Giusti, C. (2005). Methodological proposal for the assessment of the scientific quality of geomorphosites. Il Quaternario 18 (1), 303À313;

Costăchescu, N. (1906). Les gas contenus dans la sel gemme et dans les volcans de boue de la Roumanie. Anal. Şt. Univ. Iaşi, IV (1): 3-59;

Frunzescu, D. \& Branoiu, G. (2004). Monografia geologică a bazinului râului Buzău, editura Universităţii din Ploieşti, 458p;

Giusti, Christian. (2012). Les géosites d'intérêt géomorphologique ou géomorphosites dans l'inventaire du géopatrimoine.

Grecu, F. (2017). Interconditionality geomorphosites and natural hazards, in vol. Riscuri şi catastrofe, editor Sorocovschi V., anXVI, vol.20, nr.1/2017, Cluj Napoca, p.41 - 52;

Grecu F. (2018). Geomorfologie pluvio-fluvială. Teorie şi aplicații, Editura Universitară, Bucureşti;

Grecu, F., Iosif, D. (2014a). La notion du Géosite et son pertinence dans une étude sur la région des Gorges du Danube en Roumanie, Analele Universităţii Bucureşti: Seria Geografie LXIII, p178-184;

Grecu, F., Iosif, D. (2014b). The geosites from Danube Defile in Romania. The vulnerability to touristic activities. Geojournal of Tourism and Geosites. 14. 178-184;

Grecu, F., Rădulescu, C.C., Comănescu, L. (2019). Relief, paysage, géomorphosite dans le Plateau de Dobroudja du Sud Roumanie. Analele Universității Bucureşti, Geographie, LXVIII, p.5-24;

Ielenicz, M. (1984). Munții Ciucaş - Buzău. Studiu geomorfologic, Editura Academiei, Bucureşti, 148 p;

Ilieş D. C, Josan N. (2007). Preliminary contribution to the investigation of the Geosites from Apuseni Mountains (Romania). Revista de Geomorfologie 9: 53-59;

Ilieş D. C, Josan N. (2008). Some theoretical aspects regarding the genesis of geosites, GeoJournal of Tourism and Geosites Year I, no. 1, vol. 1, 2008, pag. 7-12;

Ilieş D. C., Josan N. (2009). Geosituri şi geopeisaje. Editura Universităţii din Oradea;

Institutul de Geografie (1987). Geografia României, Vol III, Carpaţii Româneşti şi Depresiunea Transilvaniei, Editura Acdemiei, Bucureşti, 656p;

Institutul de Geografie (1992). Geografia României, Vol IV, Regiunile pericarpatice, Editura Academiei, Bucureşti, 580p;

Iosif, D. (2014). La fiche d'évaluation des géosites. Un exemple exhaustif utilisé sur des sites roumains. Cinq Continents 4 (10): 158-180;

Macarovici, N. (1961). Observaţii stratigrafice pe structura BercaArbănaşi (cu privire asupra limitelor Dacianului). St. cerc. geol., VI (3): 387-403;

Mocanu-Vargancsik, C. A., \& Barbulescu, A. (2018). Analysis on Variability of Buzau River Monthly Discharges, Ovidius University Annals of Constanta - Series Civil Engineering, 20(1), 51-56;

Panizza, M., (2001). Geomorphosites: Concepts, methods and examples of geomorphological survey, Chinese Science Bulletin, 46, 4-6;

Popa, M.C. \& Peptenatu, D. \& Draghici, C. \& Diaconu, D. (2019). Flood Hazard Mapping Using the Flood and Flash-Flood Potential Index in the Buzău River Catchment, Romania. Water. 11. 2116. 10.3390/w11102116; 
Rădulescu, C. C., Grecu, F. (2017). General and particular in the inventory and assessment of geosites in southern Dobrudja, Romania. Analele Universităţii Bucureşti: Seria Geografie, 2018, p39-62;

Reynard, E. (2006). Fiche d'inventaire des géomorphosites, Université de Lausanne, Institut de géographie, rapport non publié, 8 pages;

Reynard, E. (2009). Geomorphosites: Definitions and characteristics. Geomorphosites, 9-20;

Reynard, E., Fontana G., Kozlik, L., Scapozza, C. (2007), A method for assessing «scientific» and «additional values» of geomorphosites, Geographica Helvetica Jg. 62, Heft 3, 148158 ;

Reynard, E. Coratza, P., Calya, N., Clivaz, M., Comănescu, L., Darbellay, L., Giusti, C., Grecu, F., Hobléa, F., Pereira, P. (2018). INTERGEO: A digital platform for university education on geomorphological heritage, Geoheritage, https://doi.org 10.1007/s12371018-0298-8, p.1-13;

Sencu, V. (1985). Vulcanii noroioşi de la Berca. Ed. Sport-Turism, Bucureşti, 21 pp;

Ujvari, I. (1972). Geografia apelor României, Editura ştiinţifică, Bucureşti, 592p;

Visarion M., Săndulescu, M., Drăgoescu, I., Drăghici, M., Cornea, I., Popescu, M. (1977). România. Harta mişcărilor crustale verticale recente, 1:1 000000 . Institutul de Geologie şi Geofizică, Bucureşti, p. 3-10; 
